\title{
Intermediate Stage Complex Regional Pain Syndrome Type 1 Is Unrelated to Proinflammatory Cytokines
}

\author{
Renate J. M. Munnikes, Christel Muis, Martine Boersma, Claudia Heijmans-Antonissen, \\ Freek J. Zijlstra, and Frank J. P. M. Huygen \\ Department of Anesthesiology, Pain Treatment Centre, Erasmus MC, PO Box 2040, 3000 CA Rotterdam, The Netherlands
}

Received 22 June 2005; accepted 12 August 2005

\begin{abstract}
The aim of this paper is to determine the involvement of tumour necrosis factor- $\alpha$ (TNF- $\alpha$ ) and interleukin (IL)- 6 in intermediate CRPS 1 as locally formed mediators of inflammation. In this study, 25 patients with proven CRPS 1 (Bruehl criteria) were included. All patients participated in one of our earlier studies during the acute stage of their disease. After the disease developed into an intermediate stage, both the disease activity and the profile of inflammatory mediators were reevaluated. Disease activity and impairment were determined by means of a visual analogue scale, the McGill Pain Questionnaire, the difference in volume and temperature between the involved and uninvolved extremities, and the reduction in active range of motion of the involved extremity. Suction blisters were made on the involved and uninvolved extremities for measurement of IL- 6 and TNF- $\alpha$. A significant improvement in signs and symptoms of impairment was found. However, the levels of IL- 6 and TNF- $\alpha$ in blister fluid in the involved extremity versus uninvolved extremity were still significantly raised. Although signs and symptoms are significantly improved, proinflammatory cytokines are still increased in CRPS 1 affected extremities during the intermediate stage of the disease. This indicates that the initiation and sustained development of the disease are only partially affected by proinflammatory cytokines. Follow-up in the chronic stage is necessary to draw more definite conclusions about the existence of a supposed relation between clinical signs and symptoms and the level of proinflammatory cytokines.
\end{abstract}

\section{INTRODUCTION}

The complex regional pain syndrome type 1 (CRPS 1) is a disease occurring in an extremity after an initiating noxious event, such as a Colles fracture or minor surgery, which is mainly characterised by spontaneous pain, allodynia, and hyperalgesia. Complex regional pain syndrome type 2 (CRPS 2) develops after a nerve injury. The symptoms in CRPS 1 are not limited to the region of a single peripheral nerve and are generally disproportional to the inciting event. There is (or has been) evidence of oedema, disturbed blood flow or abnormal sudomotor activity in the affected limb. CRPS 1 diagnosis is excluded by the existence of conditions that would otherwise account for the

Correspondence and reprint requests to Freek J. Zijlstra, Department of Anesthesiology, Pain Treatment Centre, Erasmus MC, PO Box 2040, 3000 CA Rotterdam, The Netherlands; f.zijlstra@erasmusmc.nl

This is an open access article distributed under the Creative Commons Attribution License which permits unrestricted use, distribution, and reproduction in any medium, provided the original work is properly cited. degree of pain and dysfunction. The pathophysiology of CRPS 1 remains unclear. Several studies suggest a peripheral afferent mechanism, others suggest a peripheral efferent mechanism, or central mechanisms including psychological mechanisms or combinations of more than one mechanism have been suggested. Evidence for inflammation was shown by Huygen et al; an increase in the levels of IL- 6 and TNF- $\alpha$ in blister fluid of the involved versus uninvolved extremity in the acute phase suggests a local inflammatory process [1].

The course of symptoms in CRPS has been poorly studied. Classic teaching is that patients afflicted with this disorder progress through three stages, but this is mainly based on clinical observation rather than scientific data [2]. In general, the disease is characterised in the acute stage by symptoms of regional inflammation, which are severe burning pain, muscle spasm, joint stiffness, restricted mobility, rapid hair and nail growth, and vasospasm that affect colour and temperature of the skin. The stage is also indicated as the "warm-" type CRPS 1, due to increased skin temperature as a result of local inflammation. In the chronic stage of the disease, changes in skin and bones become irreversible and pain spreads throughout the entire limb and a more neuropathy-like 
disorder develops. A possible explanation for this alteration during the disease is the development of sensitization or plasticity during the regional inflammation, which results in a neuropathic disorder. At that stage, blood flow and tissue-blood distribution are diminished, partly caused by disuse of the extremity, usually resulting in a decreased skin surface temperature, and therefore also indicated as the "cold-" type CRPS 1 . In the intermediate stage of the disease (1-2 years after the initial event), inflammatory mediators, neuropathic substances, and vasoactive agents could play a prominent role. In general, at that stage of the disease, marked temperature elevations have been diminished or even normalised [3].

Veldman et al did not observe any temporal trend in prospective assessment of 829 patients with reflex sympathetic dystrophy (RSD), one of the formerly used indications to describe the disease [4]. Retrospective surveys completed by CRPS patients with an average pain duration of over 3 years similarly indicate that CRPS symptoms most often tend to remain stable or even improve, rather than progressively deteriorate [5].

Since a diminution of the clinical symptoms is to be expected in a certain time course, we hypothesized that local inflammation would only be present during the initial, acute stage of the disease, and therefore the formation of proinflammatory mediators (such as IL- 6 and TNF- $\alpha$ ) should be attenuated during the course of the disease. It is interesting to know whether this decline is correlated with an improvement in disease activity as measured by registration of pain, and by the measurement of differences in temperature, volume, and mobility of the joints between the involved and uninvolved extremities.

\section{MATERIALS AND METHODS}

\section{Patients}

The study was approved by the medical ethical committee of the Erasmus Medical Centre Rotterdam.

Between April 2001 and February 2004, 66 patients with CRPS 1 participated in several studies either to investigate the pathophysiology or the effects of specific treatment of CRPS $1[1,6,7]$.

In retrospect, 6 of 66 patients proved to have CRPS 2 . Fifty nine patients, who were not involved in any study for at least 6 months, were contacted by telephone and mail to fill in a short questionnaire about their disease; 49 questionnaires were returned. These latter patients were asked to participate in a follow-up measurement. For practical reasons, only patients with CRPS 1 of one upper extremity were selected (making artificial blisters in the skin of the lower extremity is not always as easy). Patients using corticosteroids and/or immunosuppresives after the first measurement ( $\mathrm{T} 0)$ were also excluded.

Finally, 25 patients with CRPS 1 , at the time of the follow-up study all being in the so-called intermediate stage of the disease, agreed to participate. All the patients fulfiled the CRPS 1 criteria by Bruehl et al at the first measurement, performed shortly after the initial event which resulted in the development of the disease [8]. These modified diagnostic criteria include the report of at least one symptom in each of the following categories: (i) hyperesthesia, (ii) temperature asymmetry and/or skin colour changes and/or skin colour asymmetry, (iii) oedema and/or sweating changes and/or sweating asymmetry, and (iv) decreased range of motion and/or motor dysfunction (weakness, tremor, dystonia) and/or trophic changes (hair, nail, skin); and must display at least one sign in two or more of the following categories: (i) evidence of hyperalgesia (to pinprick) and/or allodynia (to light touch), (ii) evidence of temperature asymmetry and/or skin colour changes and/or skin colour asymmetry, (iii) evidence oedema and/or sweating changes and/or sweating asymmetry, and (iv) evidence of decreased range of motion and/or motor dysfunction (weakness, tremor, dystonia) and/or trophic changes (hair, nail, skin).

Signs and symptoms of impairment were assessed by registration of pain, and by the measurement of differences in temperature, volume, and mobility between the involved and uninvolved extremities. Blisters were made with a suction method in order to determine proinflammatory cytokines in the involved and uninvolved extremities.

\section{Registration of pain}

The intensity of pain was assessed using a visual analogue scale (VAS) recorded in 0-100 millimetres and the McGill Pain Questionnaire, Dutch Language Version (MPQ-DLV). The MPQ-DLV score was measured by counting the total number of words chosen from 20 items [9].

\section{Temperature}

Skin temperature was measured with an infrared tympanic probe thermometer (First Temp Genius, Sherwood Medical, Crawley, Sussex, UK). Temperature was measured on the dorsal aspect of the hand in a matrix of 5 points. The difference in mean temperature between the involved and uninvolved extremities was calculated $[1,6,7,10,11]$.

\section{Oedema}

Determination of the volume of the hands was assessed by a volumeter, which weighs the amount of water displaced by the immersion of a body part. The difference between the involved and uninvolved extremities was calculated as a percentage of the uninvolved extremity [12].

\section{Mobility}

Mobility was assessed by means of measurement of active range of motion (AROM). The AROM is defined as the arc of motion with muscle power to achieve the motion of a joint. In the upper extremity, the AROM was measured for the dorsal/palmar flexion in the wrist, for the flexion/extension in the metacarpophalangeal and 
TABle 1. Demographic data. Data are presented as median \pm interquartile range. Two patients participated in 2 studies. References: literature on reported effects in CRPS 1.

\begin{tabular}{l|l}
\hline Patient characteristics & Number \\
\hline Sex (male/female) & $6 / 19$ \\
Age at follow-up (years) & $54(49.5-57.5)$ \\
Duration of CRPS 1 (months) at first measurement & $6(2-12)$ \\
Duration of CRPS 1 (months) at follow-up & $30(23-40)$ \\
Fracture/trauma/surgery/spontaneous & $10 / 8 / 6 / 1$ \\
Treatment during the course of the disease (T0-Tf) & 12 patients with no specific treatment/physiotherapy [13] \\
& 4 patients with DMSO [14, 15] \\
& 5 patients with capsaicin [16] \\
& 1 patient with anti-TNF [7] \\
& 5 patients with acupuncture [17] \\
\hline
\end{tabular}

proximal interphalangeal joints of the two most restricted digits.

The position of the patients and the method of measurement were standardized for each joint, conforming to the American Society of Hand Therapists clinical assessment recommendations [13]. The AROM of the involved extremity was multiplied by 100 and divided by the AROM on the uninvolved extremity: this resulted in a percentage of normal mobility. The compounded percentage was then scored from 1 to 5 , indicating a percentage of mobility: score $1 \geq 95 \%$ normal mobility; $2=94 \%-85 \%$; $3=84 \%-65 \% ; 4=64 \%-25 \%$; and $5 \leq 25 \%$ normal mobility. The sum of points was calculated which could range from 5 (normal mobility) to 25 (most abnormal mobility) $[1,6,7,10]$.

\section{Blisters}

Blisters were induced by means of a suction method [11]. A 3-well skin suction chamber was positioned on the skin of the upper extremity, on the dorsal side of the involved hand and the flexor side of the uninvolved forearm.

A vacuum of $300 \mathrm{~mm} \mathrm{Hg}$ was applied with a Atmoforte 350A aspirator pump (ATMOS Medizintechnik, Lenzkirch, Germany), which was reduced after 15 minutes to $250 \mathrm{~mm} \mathrm{Hg}$ and again, 15 minutes later, reduced to $200 \mathrm{~mm} \mathrm{Hg}$. This negative pressure was maintained for 2 to 2.5 hours. The developed blisters were punctured and produced approximately $150 \mu \mathrm{l}$ blister fluid from each side. All samples were stored at $-80^{\circ} \mathrm{C}$ until analysis $[1,6,7,10,11]$.

\section{Laboratory assays}

Blister samples were diluted 4-fold in appropriate calibrator diluent assay buffer for the direct measurement of cytokines. Cytokine assays were performed following the manufacturer's protocol (Pelikine Human ELISA Kits for IL-6 (M1906) and TNF- $\alpha$ (M1920), CLB, Amsterdam, the Netherlands). The standard curve ranges and mean calculated zero signal +3 SD for IL- 6 were $0-80 \mathrm{pg} / \mathrm{mL}$ and $0.3 \mathrm{pg} / \mathrm{mL}$, respectively; and for TNF- $\alpha 0-1000 \mathrm{pg} / \mathrm{mL}$ and $1 \mathrm{pg} / \mathrm{mL}$, respectively. The absorbance per well was measured at $450 \mathrm{~nm}$ with a Medgenix ELISA reader. Sam- ple concentrations were calculated using the appropriate standard calibration lines and the Softmax software of the reader.

\section{Statistical analysis}

Because of a nonlinear distribution, the data of cytokine contents in blister fluids were described as median and interquartile ranges. Comparison of paired samples was performed with the Wilcoxon signed-ranks test. Significance was accepted with a $p<0.05$ (two-sided).

\section{RESULTS}

A group of 25 patients with an intermediate duration of CRPS 1 (median 30 months, interquartile range 23-40 months) in one extremity were examined in the follow-up of their CRPS; patient characteristics and participation in previous studies are presented in Table 1.

The signs and symptoms of impairment are described in terms of pain and differences in temperature, volume, and mobility between the involved and uninvolved extremities during the first (T0) and follow-up (Tf) measurement. Difference in volume, AROM, VAS, and McGill Pain Questionnaire show a significant decrease at Tf compared to T0 (Wilcoxon signed-ranks test, $p<0.05$ ). Despite a tendency to decrease, the temperature difference between the involved and uninvolved extremities during the first (T0) and follow-up (Tf) measurement is not significant. Data on these parameters are given in Table 2.

This study showed a significant elevation of IL-6 and TNF- $\alpha$ levels in the involved extremity compared with the uninvolved extremity in patients with intermediate CRPS 1 at follow-up as was found in the first measurement (Wilcoxon signed-ranks test, $p<0.05)[1,6,7]$. There is no significant difference in IL- 6 and TNF- $\alpha$ levels in both involved and uninvolved extremities between T0 and Tf. These data are presented in Table 3 and in Figure 1a (IL6) and Figure $1 \mathrm{~b}(\mathrm{TNF}-\alpha)$.

Three patients considered themselves to be cured of CRPS 1. These are patients 3, 10, and 24 in Figures 1a and 1b. Patient 3 showed a slight decrease in strength. Patient 10 , though considering herself as cured, still had signs of 
TABLE 2. Signs and symptoms of impairment in the study population at first measurement (T0) and follow-up measurement (Tf). Data are presented as median \pm interquartile range.

\begin{tabular}{|c|c|c|}
\hline$N=25$ & T0 & $\mathrm{Tf}$ \\
\hline $\begin{array}{l}\text { Difference in temperature between involved and uninvolved sides } \\
\left(\text { delta } \mathrm{T} \text { : in }{ }^{\circ} \mathrm{C} \text { ) }\right.\end{array}$ & $\begin{array}{c}0.2 \\
(-0.36 \text { to }+0.75)\end{array}$ & $\begin{array}{c}0.04 \\
(-0.57 \text { to }+0.34)\end{array}$ \\
\hline $\begin{array}{l}\text { Difference in volume between involved and uninvolved sides } \\
\text { (delta V: in \% volume uninvolved extremity) }\end{array}$ & $\begin{array}{c}12 \\
(-7 \text { to }+27.5) \\
\end{array}$ & $\begin{array}{c}-13 \\
(-40.5 \text { to }+37)^{*}\end{array}$ \\
\hline $\begin{array}{l}\text { Active range of motion } \\
\text { (AROM: sum of numbers which express percentage of normal } \\
\text { mobility, extremity: 5-25: normal mobility-most abnormal) }\end{array}$ & (11 to 19.5 ) & $\begin{array}{c}10 \\
(8 \text { to } 13)^{*}\end{array}$ \\
\hline $\begin{array}{l}\text { McGill Pain Questionnaire } \\
\text { (MPQ: number of counted words that describe pain) }\end{array}$ & $\begin{array}{c}11 \\
(9 \text { to } 14)\end{array}$ & $\begin{array}{c}4 \\
(2 \text { to } 8.75)^{*}\end{array}$ \\
\hline $\begin{array}{l}\text { Pain by means of visual analogue scale } \\
\text { (VAS: } 0-100 \mathrm{~mm} \text { : no pain-most pain) }\end{array}$ & $\begin{array}{c}60 \\
(46 \text { to } 72)\end{array}$ & $\begin{array}{c}40 \\
(7.5 \text { to } 65.5)^{*}\end{array}$ \\
\hline
\end{tabular}

*Wilcoxon signed-rank sum test $p<0.05$.

fibrosis and contracture in the palm of the hand. Light touch was unpleasant.

Patient 24 had only some minor hypesthesia of the affected extremity. Nine patients experienced no change in CRPS and one patient had an increase in symptoms. Twelve patients reported a decrease of the symptoms.

At physical examination, there was a general improvement of physical signs of CRPS 1 in all 25 patients.

Most patients could be considered as cured with variable light to moderate signs of contracture and weakness. Only patient 25 had severe allodynia and functional loss combined with a decrease in temperature of the involved hand.

\section{DISCUSSION}

The involvement of the immunologic system in CRPS is still a matter of debate. Treatment with radical scavengers such as dimethylsulfoxide (DMSO), which in some cases proved to be beneficial, suggested the contribution of an initially occurring inflammatory reaction $[14,18]$. Our research group found evidence of the involvement of the immunologic system by the presence of proinflammatory cytokines in blister fluid collected in patients with (sub-)acute CRPS 1 [1]. This may cause activation and sensitization of primary afferents and, by this mechanism, neuropeptide release in the affected body region (mainly, substance P (SP) and calcitonin gene-related peptide (CGRP)), thereby resulting in a neurogenic inflammation [19].

In intermediate and long-term CRPS 1 patients, there is no indication of the involvement of these immunologic indices reflected in blood samples [20, 21]. In general, the sequence of (inflammatory) mediators released during the subsequent processes will result in a cascade of distinguishing substances which might influence each other's production. The sequential production of neuropeptides, cytokines, chemokines, eicosanoids, nitric oxide, and other vasoactive substances is dependent on cells involved at certain time intervals of the ongoing process and crosstalk between migrated cells $[3,18]$. So far, limited information is available concerning representative parameters reflecting consecutive stages of this disease. Although in general a warm CRPS 1 is observed during the initial stage of the disease, not all patients turn into cold CRPS 1 from the intermediate to the chronic stage of the disease. Assuming that skin surface temperature not only reflects tissue-blood distribution but also local inflammation, based on videothermographic measurements of the CRPS1 extremity (3), after the initial inflammatory event, a diminished amount of (inflammatory) mediators present in the interstitial fluid would have been expected during the intermediate stage of the disease.

During this stage of the disease, some signs and symptoms, such as volume of the extremity, AROM, McGill Pain Questionnaire, and VAS pain are significantly improved (Table 2), suggesting a spontaneous or treatmentinitiated recurrence of the disease severity. Although a slight decline of proinflammatory cytokines is observed, these mediators are still increased in CRPS 1 affected extremities (Table 3, Figures 1 and 2). This indicates that the initiation and sustained development of the disease is at least partially affected by proinflammatory cytokines. Differences in mean temperature during the first measurement and the follow-up measurement were not significantly decreased. Furthermore, characteristic indicators of skin surface temperature were not directly related to blister fluid levels of the cytokines IL- 6 and TNF- $\alpha$. This suggests a complex mechanism in which a number of cells and mediators play a role. At this stage, the most prominent cell source to generate these cytokines could be monocytes, tissue macrophages, and mast cells [6]. Although the cell counts will not dramatically change during this stage of the disease, both the activation and the cell-cell interaction could have changed, due to fluctuating levels of neuropeptides. On the other hand, long-term disuse or immobility will certainly affect the accumulation of peptides secreted by the nerves, which could contribute 
TABLe 3. Data on IL-6 and TNF- $\alpha$ levels in blister fluid in the involved and uninvolved extremities at first measurement (T0) and follow-up measurement (Tf). Data are presented as median \pm interquartile range.

\begin{tabular}{|c|c|c|c|c|}
\hline$N=25$ & \multicolumn{2}{|c|}{ T0 } & \multicolumn{2}{|c|}{$\mathrm{Tf}$} \\
\hline IL-6 (pg/mL) & $\begin{array}{l}\text { Uninvolved } \\
3(1.7-18.5)\end{array}$ & $\begin{array}{c}\text { Involved } \\
41(7.5-98)^{*}\end{array}$ & $\begin{array}{l}\text { Uninvolved } \\
2.1(1.2-4.9)\end{array}$ & $\begin{array}{c}\text { Involved } \\
24.3(3.5-165.6)^{*}\end{array}$ \\
\hline TNF- $\alpha(\mathrm{pg} / \mathrm{mL})$ & $5.9(3.1-32.5)$ & $26(12-85)^{*}$ & $7.8(5.1-16.7)$ & $31.6(7.7-90.4)^{*}$ \\
\hline
\end{tabular}

*Wilcoxon signed-rank sum test $p<0.05$.

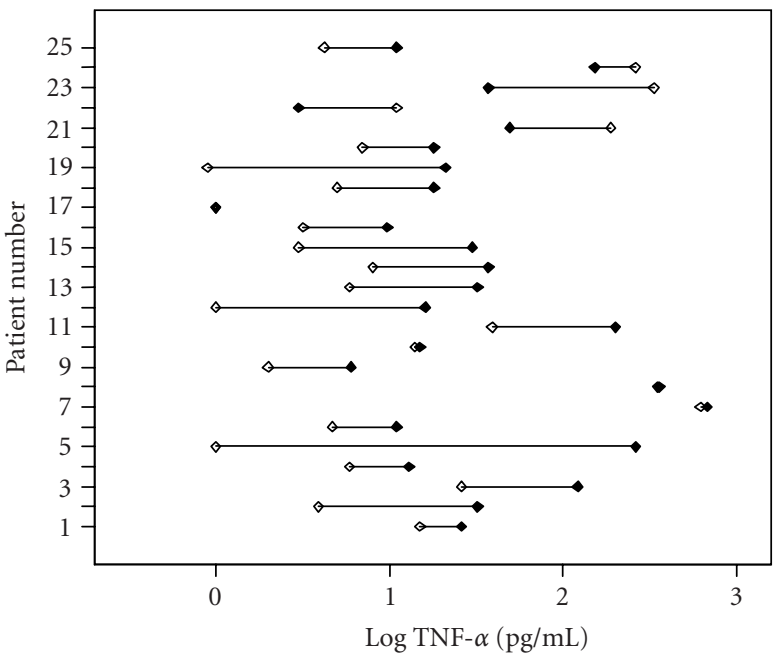

(a)

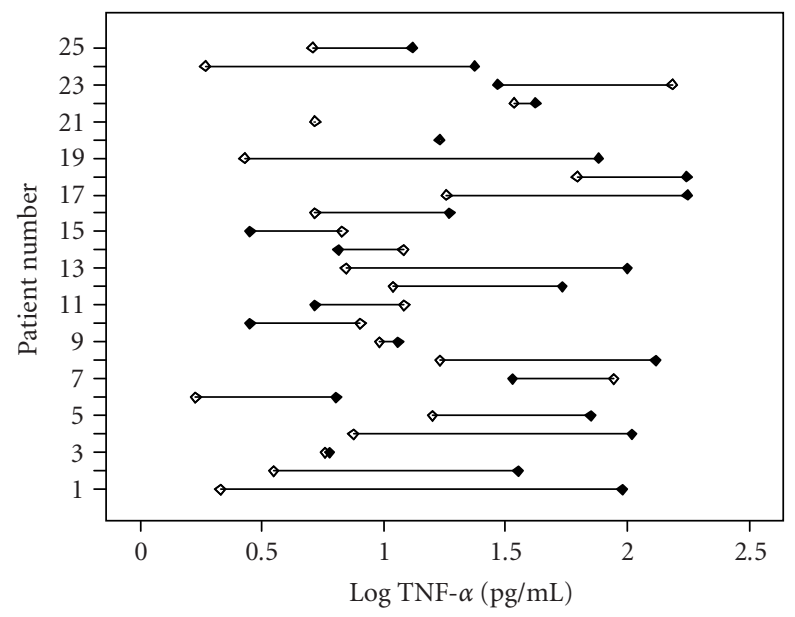

(b)

FIGURE 1. Individually plotted values of TNF- $\alpha$ (logarithmic scale) in involved (closed symbols) and uninvolved sides (open symbols) of all CRPS 1 patients, (a) at first measurement (T0) and (b) at follow-up measurement (Tf). Note that on T0 (acute stage), 19 patients displayed an increased value in the involved versus the uninvolved extremity, whereas on $\mathrm{Tf}$ (intermediate stage), still 16 patients displayed an increased value.

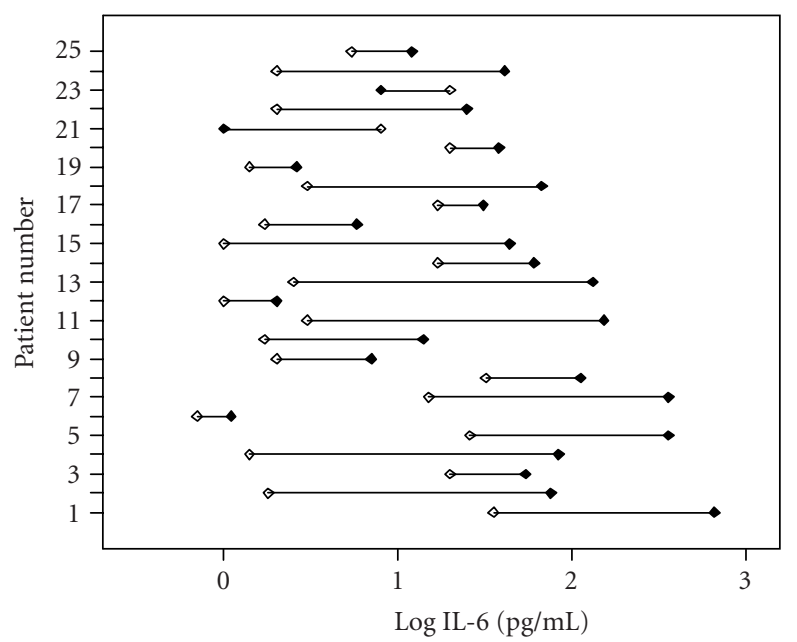

(a)

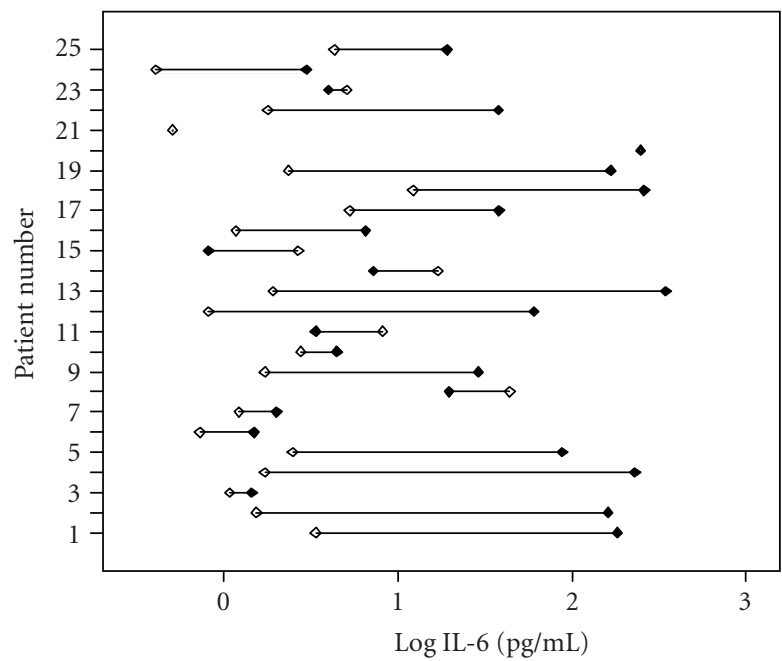

(b)

FIGURE 2. Individually plotted values of IL-6 (logarithmic scale) in involved (closed symbols) and uninvolved sides (open symbols) of all CRPS 1 patients, (a) at first measurement (T0) and (b) at follow-up measurement (Tf). Note that on T0 (acute stage), 23 patients displayed an increased value in the involved versus the uninvolved extremity, whereas on $\mathrm{Tf}$ (intermediate stage), still 18 patients displayed an increased value. 
to allodynia and hyperalgesia, but also vasomotor, sudomotor, and motor/trophic signs and symptoms.

In our results, both the ranges of data found and the abnormal distribution of cytokine levels in involved and involved extremities are remarkable (Figures 1 and 2). Although all patients were accurately included according to the criteria of by Bruehl et al [8], we cannot entirely exclude contamination of our study population. Inclusion of subpopulations which fulfil the main criteria could also influence the outcome of the results $[22,23]$.

A further follow-up of our selected CRPS 1 patients from the intermediate stage into the chronic stage (greater than 4 years) is therefore needed to draw more definite conclusions about the existence of a supposed relation between clinical signs and symptoms and local levels of proinflammatory cytokines in interstitial fluid. Furthermore, an extension of the tested pallet of cytokines should be considered in order to unravel the contribution of specific cytokines (pro- and anti-inflammatory cytokines, Th1/Th2 cell-derived cytokines, chemokines) during the whole course of the disease. More insight in the timerelated contribution of specific cytokines will enable the selective use of immunosuppressives $[7,18]$ or the specific development of disease-modifying agents.

\section{ACKNOWLEDGMENTS}

This study was performed within TREND (TraumaRelated Neuronal Dysfunction), a knowledge consortium that integrates research on complex regional pain syndrome type 1 . The project is supported by a Dutch Government Grant (BSIK03016). The help of Sjoerd Niehof (Engineer in physics) and Liesbeth Lagendijk (Research nurse) was highly appreciated. The authors thank Laraine Visser-Isles (Department of Anesthesiology) for her editorial assistance.

\section{REFERENCES}

[1] Huygen FJ, De Bruijn AG, De Bruin MT, Groeneweg JG, Klein J, Zijlstra FJ. Evidence for local inflammation in complex regional pain syndrome type $1 . \mathrm{Me}$ diators Inflamm. 2002;11(1):47-51.

[2] Bonica J. The Management of Pain. 2nd edition. Philadelphia, USA: Lea \& Febiger; 1990.

[3] Huygen FJ, Niehof S, Klein J, Zijlstra FJ. Computerassisted skin videothermography is a highly sensitive quality tool in the diagnosis and monitoring of complex regional pain syndrome type I. Eur J Appl Physiol. 2004;91(5-6):516-524.

[4] Veldman PH, Reynen HM, Arntz IE, Goris RJ. Signs and symptoms of reflex sympathetic dystrophy: prospective study of 829 patients. Lancet. 1993;342(8878):1012-1016.

[5] Galer BS, Henderson J, Perander J, Jensen MP. Course of symptoms and quality of life measure- ment in complex regional pain syndrome: a pilot survey. J Pain Symptom Manage. 2000;20(4):286292.

[6] Huygen FJ, Ramdhani N, van Toorenenbergen A, Klein J, Zijlstra FJ. Mast cells are involved in inflammatory reactions during complex regional pain syndrome type 1. Immunol Lett. 2004;91(2-3):147-154.

[7] Huygen FJ, Niehof S, Zijlstra FJ, van Hagen PM, van Daele PL. Successful treatment of CRPS 1 with antiTNF. J Pain Symptom Manage. 2004;27(2):101-103.

[8] Bruehl S, Harden RN, Galer BS, et al. External validation of IASP diagnostic criteria for complex regional pain syndrome and proposed research diagnostic criteria. International association for the study of pain. Pain. 1999;81(1-2):147-154.

[9] Lowe NK, Walker SN, MacCallum RC. Confirming the theoretical structure of the McGill pain questionnaire in acute clinical pain. Pain. 1991;46(1):53-60.

[10] Oerlemans HM, Oostendorp RA, de Boo T, Goris RJ. Pain and reduced mobility in complex regional pain syndrome I: outcome of a prospective randomised controlled clinical trial of adjuvant physical therapy versus occupational therapy. Pain. 1999;83(1):7783.

[11] Michiels JJ, Zijlstra F. Prostaglandin cyclooxygenase products but not thromboxane A2 are involved in the pathogenesis of erythromelalgia in thrombocythaemia. Mediators Inflamm. 1993;2(5):385-389.

[12] Fereidoni M, Ahmadiani A, Semnanian S, Javan M. An accurate and simple method for measurement of paw edema. J Pharmacol Toxicol Methods. 2000;43(1):11-14.

[13] Kemler MA, Rijks CP, de Vet HC. Which patients with chronic reflex sympathetic dystrophy are most likely to benefit from physical therapy? J Manipulative Physiol Ther. 2001;24(4):272-278.

[14] Zuurmond WW, Langendijk PN, Bezemer PD, Brink $\mathrm{HE}$, de Lange JJ, van Loenen AC. Treatment of acute reflex sympathetic dystrophy with DMSO $50 \%$ in a fatty cream. Acta Anaesthesiol Scand. 1996;40(3):364-367.

[15] Perez RS, Zuurmond WW, Bezemer PD, et al. The treatment of complex regional pain syndrome type I with free radical scavengers: a randomized controlled study. Pain. 2003;102(3):297-307.

[16] Ribbers GM, Stam HJ. Complex regional pain syndrome type I treated with topical capsaicin: a case report. Arch Phys Med Rehabil. 2001;82(6):851-852.

[17] Zijlstra FJ, van den Berg-de Lange I, Huygen FJ, Klein J. Anti-inflammatory actions of acupuncture. Mediators Inflamm. 2003;12(2):59-69.

[18] Huygen FJ, de Bruijn AG, Klein J, Zijlstra FJ. Neuroimmune alterations in the complex regional pain syndrome. Eur J Pharmacol. 2001;429(1-3):101-113.

[19] Birklein F, Schmelz M, Schifter S, Weber M. The 
important role of neuropeptides in complex regional pain syndrome. Neurology. 2001;57(12):2179-2184.

[20] Ribbers GM, Oosterhuis WP, van Limbeek J, de Metz M. Reflex sympathetic dystrophy: is the immune system involved? Arch Phys Med Rehabil. 1998;79(12):1549-1552.

[21] van de Beek WJ, Remarque EJ, Westendorp RG, van Hilten JJ. Innate cytokine profile in patients with complex regional pain syndrome is normal. Pain. 2001;91(3):259-261.

[22] Bruehl S, Harden RN, Galer BS, Saltz S, Backonja M, Stanton-Hicks M. Complex regional pain syndrome: are there distinct subtypes and sequential stages of the syndrome? Pain. 2002;95(1-2):119-124.

[23] Bruehl S, Chung OY, Burns JW. Differential effects of expressive anger regulation on chronic pain intensity in CRPS and non-CRPS limb pain patients. Pain. 2003;104(3):647-654. 


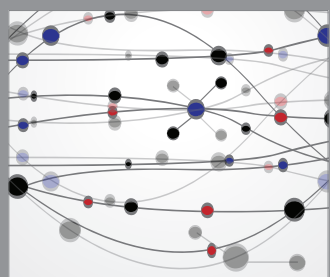

The Scientific World Journal
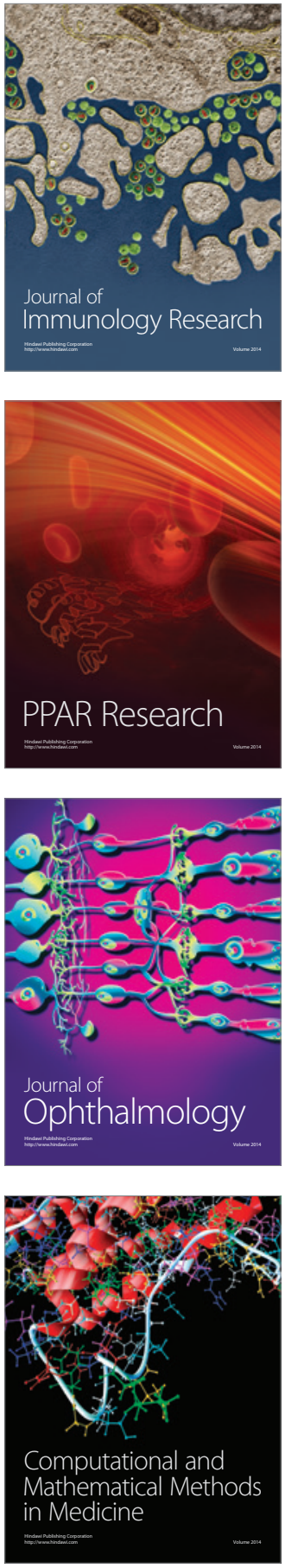

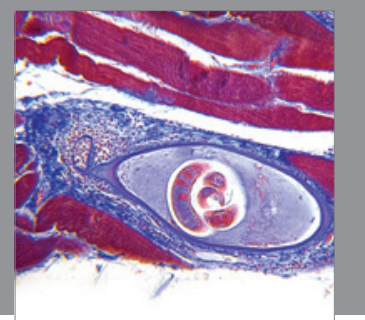

Gastroenterology

Research and Practice
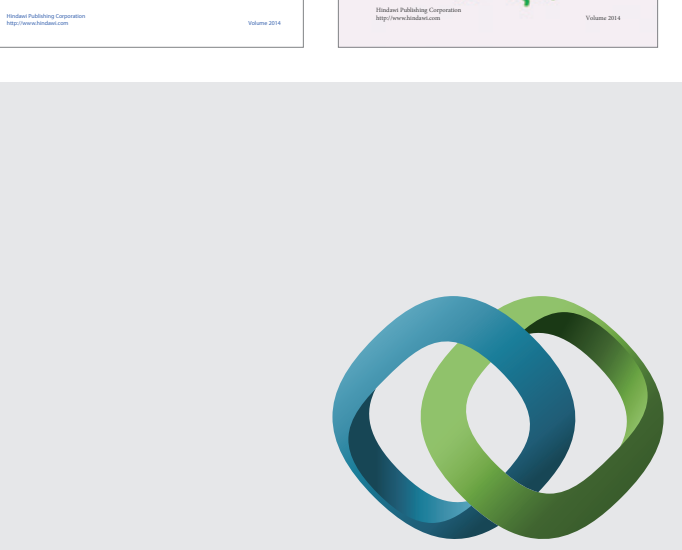

\section{Hindawi}

Submit your manuscripts at

http://www.hindawi.com
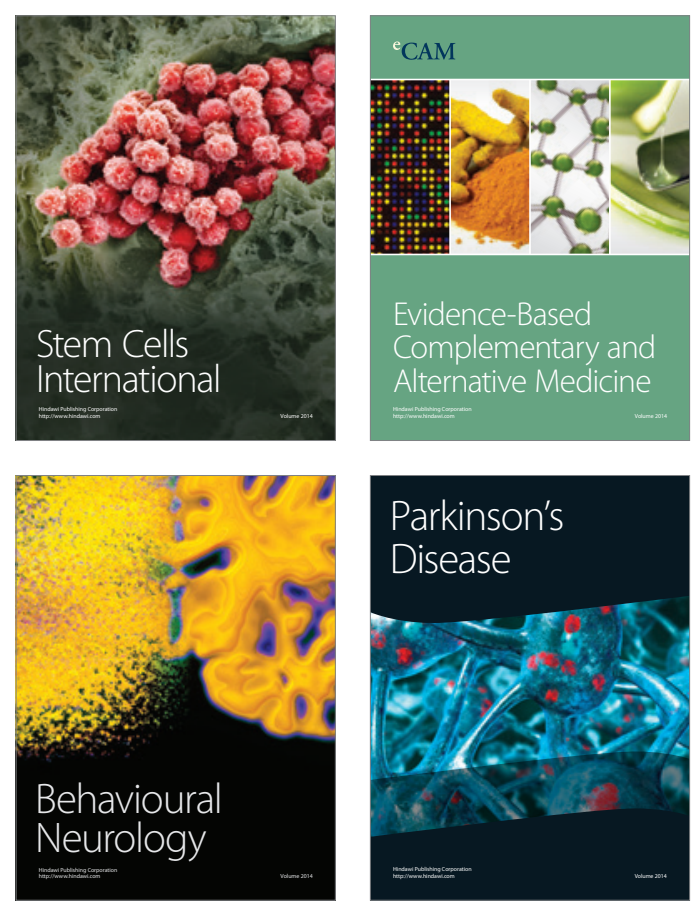

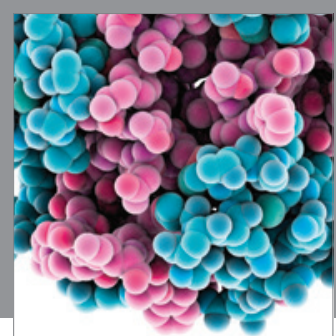

Journal of
Diabetes Research

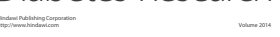

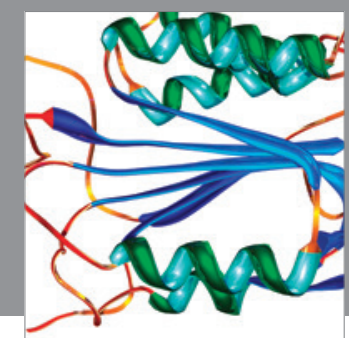

Disease Markers
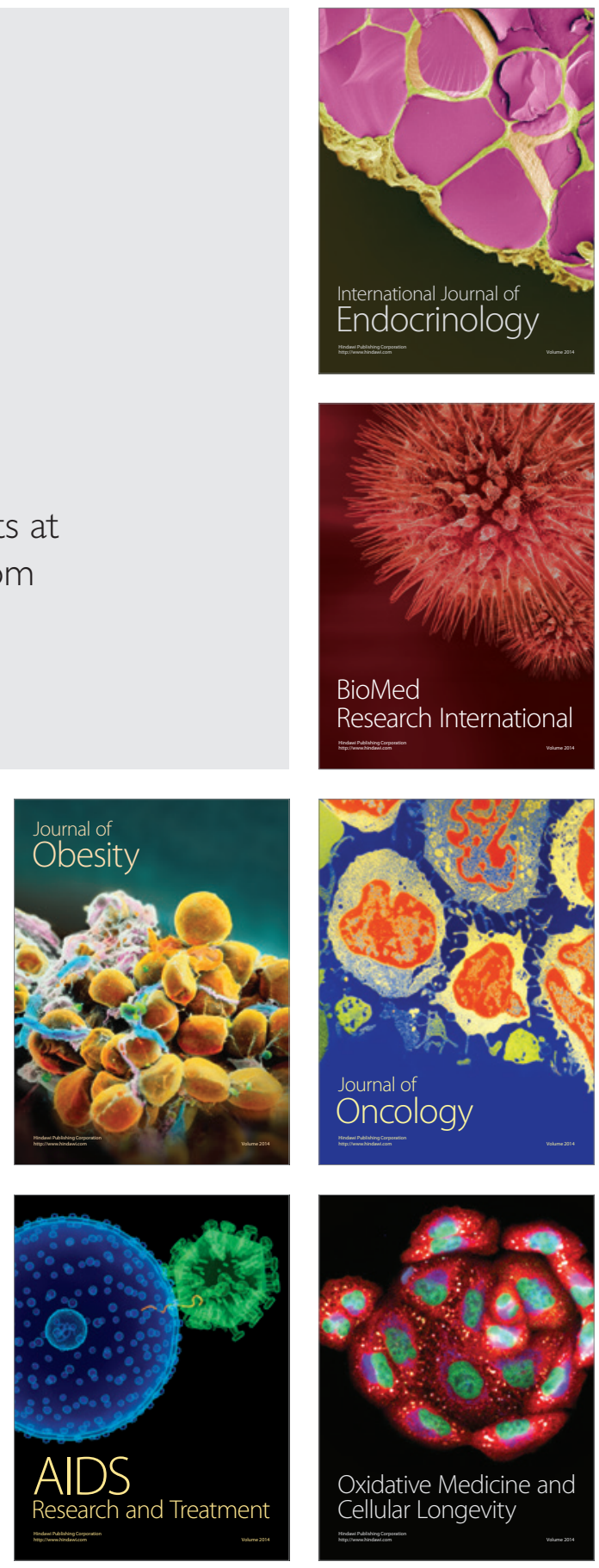